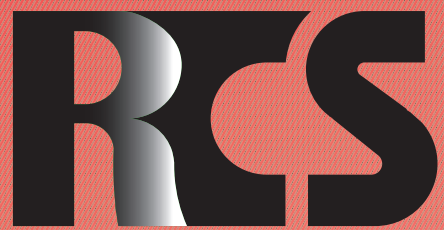

Depósito legal ppi $201502 Z U 4662$

Esta publicación científica en formato digital es continuidad de la revista impresa Depósito Legal: pp $197402 Z U 789$

- ISSN: 1315-9518 • ISSN-E: 2477-9431

Revista de Ciencias Sociales

Universidad del Zulia. Revista de la Facultad de Ciencias Económicas y Sociales Vol. XXVII. No. 2

Abril-Junio 2021

Esta publicación científica en formato digital es continuidad de la revista impresa Depósito Legal: pp $197402 Z$ Z789 ISSN: 1315-9518 


\title{
Planificación territorial en Chile: Del modelo Top Down a los desafíos de articulación multinivel
}

\author{
Montecinos, Egon*
}

\section{Resumen}

Tradicionalmente la planificación territorial y urbana en Chile se ha desarrollado bajo la tutela del centralismo, con enfoques sectoriales y poco integrales. A comienzos del año 2014, se realizó un esfuerzo sustantivo para avanzar hacia modelos de planificación horizontales, multi escalares y participativos, a través de la promulgación de la política nacional de desarrollo urbano. El propósito del artículo es conocer la opinión de actores expertos sobre los instrumentos de planificación urbana territorial, contrastarlos con los propósitos que persigue la política, y con algunas experiencias desarrolladas en región, especialmente en lo referido al rol de los gobiernos subnacionales y la sociedad civil. Para ello, se describe el diseño institucional de la planificación territorial, se exponen los resultados sobre el caso Chileno, y se analizan los factores relevantes del funcionamiento de las políticas de desarrollo urbano. Entre los principales resultados se destaca que, a pesar de que existen casos aislados de planificación urbana que se construyen de abajo hacia arriba, multiescalar y que la política de desarrollo urbano ha significado un avance, la práctica generalizada de las instituciones impide que se expandan. Como consecuencia, sigue predominando un modelo de planificación top down, sectorial y con escasa participación de actores locales.

Palabras clave: Planificación territorial; gobernanza multiescalar; participación ciudadana; gobiernos locales; ciudades.

Doctor en Investigación en Ciencias Sociales, mención Ciencia Política. Docente de la Universidad Austral de Chile, Chile. E-mail: egon.montecinos@uach.cl iD ORCID: https://orcid.org/0000-0003-4209-4351 


\title{
Territorial planning in Chile: From the Top Down model to the challenges of multilevel articulation
}

\begin{abstract}
Traditionally, territorial and urban planning in Chile has been developed under the tutelage of centralism, with sectoral and not very comprehensive approaches. At the beginning of 2014, a substantive effort was made to move towards horizontal, multi-scalar and participatory planning models, through the enactment of the national urban development policy. The purpose of the article is to know the opinion of expert actors on the instruments of territorial urban planning, to contrast them with the purposes pursued by the policy, and with some experiences developed in the region, especially with regard to the role of subnational governments and civil society. . For this, the institutional design of territorial planning is described, the results of the Chilean case are presented, and the relevant factors of the operation of urban development policies are analyzed. Among the main results, it stands out that, despite the fact that there are isolated cases of urban planning that are built from the bottom up, multiscale and that the urban development policy has meant progress, the generalized practice of the institutions prevents them from expanding. As a consequence, a top-down, sectoral planning model continues to predominate, with little participation of local actors.
\end{abstract}

Keywords: Territorial planning; multiscale governance; citizen participation; local governments; cities.

\section{Introducción}

Los acelerados procesos de urbanización han obligado a formular respuestas acordes al desarrollo de lo que se ha venido a llamar una "nueva política urbana", que implica un enfoque más integral, con objetivos plurales y articulación de actores en territorios diversos (UN-Habitat, 2014; 2017; Organización para la Cooperación y el Desarrollo Económico [OCDE], 2017; Gutiérrez, 2017). Esto ha ido configurando lo que algunos autores han llamado el policy frame para las políticas urbanas (UN-Habitat. 2017; Navarro-Yáñez y Rodríguez-García, 2020), las cuales consisten en un conjunto de orientaciones basadas en el desarrollo urbano, promovidas por la Organización de Naciones Unidas (ONU) Hábitat y los Objetivos del Desarrollo Sostenible 2030, entre otros (García, Aldape y Esquivel, 2020).

Este marco, o esquema de políticas públicas, consiste en la formulación de orientaciones genéricas sobre los objetivos y ciertas preferencias sobre instrumentos a utilizar, considerando la variable territorial, un factor esencial para alcanzar sus propósitos. La complejidad de los problemas urbanos y territoriales requiere de respuestas de políticas públicas que superen los clásicos enfoques sectoriales y que consideren de manera activa a los distintos niveles de gobierno, con un enfoque multiescalar (Rodríguez-García y Navarro, 2016; Navarro, Rodríguez-García y Ramírez-Gómez, 2018; Alencastro, et al., 2020).

Cada realidad local y regional, urbana o rural, influye en las características que adoptan los problemas que se producen $\mathrm{y}$ acogen formas concretas en contextos específicos, presentando oportunidades y capacidades que son necesarios de considerar e involucrar para elaborar políticas públicas sostenibles (Rodríguez-García y Navarro, 
2016; Navarro, et al., 2018). Es por esta razón que la planificación territorial se debe plantear de forma transversal a diferentes sectores de política pública, para atender al carácter complejo de los problemas urbanos, con objetivos integrales. Se deben desarrollar procesos de gobernanza multinivel, que permitan coordinar las actuaciones de diferentes niveles de gobierno, lo cual requiere, reconocer el carácter multi-escalar de los problemas urbanos.

Otro elemento esencial, es la participación de los actores locales, tanto agentes socio-económicos, como organizaciones sociales y la ciudadanía en general. Dichas condiciones van dando forma al desarrollo de una lógica bottom-up en el diseño e implementación de las iniciativas públicas, para garantizar que se establezcan proyectos o iniciativas que respondan a la realidad del entorno socio-espacial específico en el que se desarrollan (Quispe, Ayaviri y Maldonado, 2018).

En ese sentido, este artículo responde a la siguiente pregunta: ¿Se ajustan estos rasgos a los instrumentos de planificación territorial y a los proyectos de desarrollo urbano? Esta cuestión cobra relevancia dado que Chile, sobre todo en la última década, ha impulsado una serie de medidas para descentralizar el poder, fortalecer la participación ciudadana en la gestión pública y hacer más pertinente los instrumentos de planificación territorial a las demandas ciudadanas. Es así, que en el año 2014, se comenzó a implementar la política nacional de desarrollo urbano, y a impulsar reformas a la descentralización para asignarles mayores responsabilidades a los gobiernos subnacionales (Giménez y Ugarte, 2014).

Con el propósito de responder a esta pregunta, se tomaron en consideración los resultados de la investigación académica desarrollada en el marco de la colaboración realizada por el Centro de Estudios Regionales de la Universidad Austral de Chile a la Universidad Pablo de Olavide de España, quien lideró el proyecto titulado: "La Calidad del Diseño de la Planificación Territorial. Instrumentos para la capacitación y transferencia en la evaluación del diseño de políticas de intervención en espacios urbanos en el marco de la Nueva Agenda Urbana"(1). Dicho proyecto fue ejecutado en Perú, Ecuador, Argentina, Chile y Colombia, pero en este artículo sólo se consideran los resultados para el caso Chileno.

\section{Contexto institucional: Sistemas de gobierno local y modelos de planificación}

Chile es un país unitario y centralizado en los ámbitos político, administrativo y fiscal. En comparación con otros países unitarios, cuenta con un gasto público altamente centralizado, pues el $14,5 \%$ es ejecutado por gobiernos subnacionales versus el $27,4 \%$ promedio en la OCDE. Algo similar se verifica para la recaudación fiscal, ya que el $16,5 \%$ es a nivel subnacional versus el $28,9 \%$ promedio en la OCDE (Irarrázaval, et al., 2020)

Otro ejemplo de centralismo se aprecia en el ámbito político, puesto que hasta el año 2020, junto con Turquía, es el único país OCDE que no elige sus autoridades intermedias o regionales. Para el caso Chileno, la principal autoridad regional llamada Intendente, es designada por la presidencia de la república y esta autoridad cuenta con atribuciones políticas y fiscales responsables del gasto fiscal y coordinación política a nivel regional (Montecinos, 2005; Ortiz y Valenzuela, 2018; Montecinos, 2020).

Para el año 2021, se tiene contemplado elegir (por primera vez en la historia política del país), a la autoridad regional que reemplazará al Intendente, el que pasará a llamarse Gobernador Regional. A pesar de este importante avance, el centralismo seguirá con un representante en el nivel regional que se llamará Delegado Presidencial Regional. Esta autoridad designada, seguirá teniendo atribuciones administrativas, especialmente en lo que se refiere a la coordinación de servicios públicos nacionales y ministerios con representación en regiones, muchos 
de los cuales cuentan con instrumentos de planificación nacionales con implicancia directa en el desarrollo urbano, a escala regional y comunal.

En materia de instituciones subnacionales y sus instrumentos de planificación, el país cuenta con instituciones políticas a nivel nacional, regional y local. A escala subnacional existen 16 gobiernos regionales y 345 municipalidades (la actual Constitución de la República no reconoce a las municipalidades como gobiernos locales). En el nivel regional y local, los principales instrumentos de planificación son la Estrategia Regional de Desarrollo (ERD) y el Plan de Desarrollo Comunal (PLADECO), respectivamente.

A ellos se agregan los instrumentos de planificación del ordenamiento territorial, que a nivel regional se les denomina Plan Regional de Ordenamiento Territorial (PROT), los que a su vez se orientan por un plan nacional de ordenamiento territorial. Cada PROT debe velar y contemplar las particularidades regionales en materia territorial, con el fin de definir zonas de protección, inversión, desarrollo y crecimiento territorial. A nivel comunal se encuentran los Planes Reguladores Comunales, que establecen a nivel local las distintas zonas para el desarrollo y expansión de obras urbanas.

Junto a estos instrumentos de planificación regional y local, se encuentran las planificaciones denominadas "sectoriales" y que representan a los ministerios nacionales en regiones. Por ejemplo, los planes del Ministerio de Obras Públicas se proyectan a 20 o 30 años en el país y orientan en regiones (a través de la Secretaría Regional Ministerial de Obras Públicas) la inversión en materia de caminos, caletas pesqueras, agua potable rural, saneamiento sanitario, entre otros aspectos. En general, son proyectos muy importantes en materia de conectividad así como de desarrollo urbano para las regiones y comunas, y en promedio, su inversión dobla a la inversión total que se hace a través del gobierno regional.

Cada ministerio a nivel nacional y en su respectiva representación regional (en Chile son 23 ministerios) actúa con instrumentos de panificación que no se coordinan o articulan adecuadamente, en función de las realidades regionales o con los organismos subnacionales. Por ejemplo, a la antigua Ley General de Urbanismo y Construcciones se suman hoy al menos otras 30 leyes que afectan directamente el territorio. En específico, las facultades relacionadas con la temática de "lo urbano" y territorial, están dispersas en numerosas instituciones públicas, que se distribuyen entre el Ministerio de Vivienda y Urbanismo, Medio Ambiente, Obras Públicas, Gobiernos Regionales y Municipalidades.

Esta dispersión normativa e institucional dificulta la implementación de proyectos e iniciativas que persiguen mejoras urbanas. De acuerdo con lo planteado en los fundamentos de la Política Nacional de Desarrollo Urbano (PNDU), la cobertura de los Instrumentos de Planificación Territorial de nivel local alcanza al $68 \%$ de las comunas del país (Giménez y Ugarte, 2014).

Este número que puede parecer aceptable, esconde una realidad que afecta a muchas municipalidades y es que, por ejemplo, el proceso de elaboración y/o actualización de un plan regulador comunal, se vuelve un proceso extremadamente complejo, llevando algunas municipalidades muchos años en esta etapa de actualización. A nivel país, dichos instrumentos tardan en promedio seis años en tramitarse y tienen pocas facultades para conducir el desarrollo urbano, o para vincular los usos de suelo con el transporte, o las obras públicas con el financiamiento (Giménez y Ugarte, 2014).

Comprender este diseño institucional es muy relevante, porque permite identificar los incentivos que tienen los actores subnacionales y determina todo el recorrido y entramado burocrático que enfrenta la panificación urbana y territorial en Chile. Por ejemplo, dado que los gobiernos regionales tienen baja capacidad de gasto y generación de ingresos, estos instrumentos de planificación local y regional no tienen asegurado su financiamiento por la institución que los formula.

Es así, como un municipio para 
implementar su Plan de Desarrollo Comunal, no cuenta con los recursos municipales para cumplir con su contenido. Como consecuencia, debe recurrir a fondos regionales y sectoriales para ello $\mathrm{y} / \mathrm{o}$ hacer coincidir fondos $\mathrm{o}$ programas de financiamiento regional o sectorial con su plan de desarrollo local. Similar situación sucede con los gobiernos regionales, los cuales no cuentan con los recursos regionales suficientes para financiar las iniciativas contempladas en la Estrategia Regional de Desarrollo, debiendo recurrir necesariamente a fondos y recursos sectoriales con el fin de cumplir con lo definido en sus contenidos.

Este diseño no se modificará completamente con la reforma política a regiones que impactará al nivel de gobierno regional y comunal. Tal como se indicó, a partir del año 2021 el nuevo gobierno regional lo constituirá el gobernador y el consejo regional. El gobernador regional, será la autoridad electa y ejercerá las funciones de presidente del consejo regional y deberá coordinar así como fiscalizar a los servicios públicos que dependan o se relacionen con el gobierno regional (Montecinos, 2020).

No obstante, bajo la coordinación del Gobernador Regional no quedarán los representantes de los ministerios en regiones, llamados Secretarios Regionales Ministeriales (SEREMIS), autoridades encargadas de implementar los instrumentos de planificación sectoriales. Estas autoridades sectoriales en regiones, quedarán bajo la coordinación de la autoridad designada llamada "delegado presidencial regional".

De esta manera, las competencias y funciones exclusivas del gobernador regional serán la formulación de la estrategia regional de desarrollo, el plan regional de ordenamiento territorial, los planes reguladores metropolitanos e intercomunales, pero no tendrá tutela administrativa sobre las planificaciones sectoriales de los ministerios que tienen implicancia regional.

En definitiva, y de acuerdo con este diseño institucional, se puede señalar que en Chile existe un modelo de administración y no de gobierno tanto en el nivel regional como en el local, el cual posee características similares a las ideas planteadas en el modelo de Gobierno local limitado (Navarro-Yáñez y Rodríguez-García, 2020). Este modelo se caracteriza por la combinación de cierto nivel de capacidades políticas, pero con un bajo nivel de capacidades administrativas, en un marco de dependencia fiscal de administraciones supra-municipales, que para el caso chileno se trata del nivel regional (en relación a los municipios) y nacional-sectorial (en relación a regiones y municipios).

Coincide con lo sostenido por algunos autores, quienes señalan que la región latinoamericana muestra que sus rasgos se acercan a los del modelo de sistema de gobierno local limitado, pero jugando un papel más residual en lo que se refiere a sus capacidades administrativas (Navarro y Ramírez, 2000; Ruano y Vial, 2016; Grin, et al., 2019). En algunas municipalidades, sobre todo las de mayor tamaño y capacidad administrativa y fiscal (como las capitales regionales), también podría parecerse al modelo de "agencia local" donde el municipio se transforma en un agente implementador de políticas supra-municipales, y cuentan para ello con capacidades administrativas, pero en el marco de un bajo nivel de capacidades políticas.

En cuanto al modelo de planificación predominante, se puede apreciar una planificación sectorial top down, de arriba hacia abajo, con un fuerte predominio de las instituciones nacionales sectoriales en la elaboración/diseño de políticas públicas, $\sin$ enfoque territorial, con una incipiente articulación e integración con niveles de gobierno subnacional, escasa participación de la ciudadanía en su elaboración, diseño e implementación (Orellana, Vicuña y Moris, 2017).

En los instrumentos de planificación subnacionales, entiéndase por ellos los de los gobiernos regionales y las municipalidades, se puede apreciar un mayor protagonismo de la sociedad civil en la implementación/ ejecución de políticas y planes. En ellos se 
aprecia una mirada con enfoque territorial y la participación ciudadana se incorpora con muchas más intensidades, dinámicas y formas en los instrumentos de planificación del nivel regional y local. En general, la participación ciudadana de distintos actores territoriales se observa mucho más en la elaboración de estos instrumentos de planificación subnacional, decayendo en la etapa de implementación y ejecución (Montecinos, 2006; Delamaza, 2011; De La Fuente y Mlynarz, 2013).

\subsection{El desafío de las nuevas políticas urbanas: La respuesta gubernamental a través de la política nacional de desarrollo urbano}

Ante este diagnóstico, en abril del año 2012, se conformó una Comisión Asesora Presidencial para formular una Política Urbana que fuera capaz de guiar el desarrollo futuro de las ciudades y comunas de Chile. Con dicho hito, se dio inicio a un trabajo de participación amplia de actores sociales, expertos, académicos y autoridades políticas, para que realicen sus aportes desde los distintos ámbitos de actuación pública. El principal producto fue la promulgación de una nueva Política Nacional de Desarrollo Urbano para Chile (Giménez y Ugarte, 2014). El objetivo estratégico era contar con un instrumento de planificación nacional, con la finalidad de proyectar con optimismo el futuro de las ciudades y confiar en la implementación y proyección de esta política urbana más allá de un particular período presidencial.

¿Cuáles son los propósitos de esta política? El propósito general es alcanzar una mejor calidad de vida para las personas, abordando de manera integral los aspectos que rigen la conformación de las ciudades, propiciar la descentralización del país, acercando las decisiones de carácter local a las personas, proveer un marco explícito que posibilite una reorganización institucional y ordene el accionar de los diversos organismos y actores públicos así como privados que intervienen en las ciudades y el territorio, evitando acciones contradictorias o descoordinadas (Giménez y Ugarte, 2014; Livert, Gainza y Mogollón, 2018).

En consecuencia, los principios por los cuales se rige esta política de desarrollo urbano son la gradualidad, descentralización, equidad, integración social, participación, identidad, compromiso, calidad, eficiencia, adaptabilidad, resiliencia y la seguridad. Se organiza en los siguientes ámbitos temáticos: Integración social, desarrollo económico, equilibrio ambiental, identidad y patrimonio, $\mathrm{y}$, como requisito para el cumplimiento de los objetivos planteados en dichos ámbitos, institucionalidad y gobernanza.

¿Quérol juegan los niveles subnacionales en el marco de esta política? El rol asignado se puede identificar claramente en el ámbito de la Institucionalidad y Gobernanza Urbana a través de la promoción del cumplimiento de diversos objetivos. En general, se sostiene que para la materialización de la política, es fundamental efectuar un reordenamiento institucional, tanto de la administración central como de los gobiernos locales para avanzar hacia una mayor articulación multinivel.

Con dicho reordenamiento los objetivos y lineamientos expresados tendrán mayores opciones de materializarse, dado que no basta con mejorar la coordinación, sino que se debe avanzar hacia procesos integrados, planificados, descentralizados y participativos. Esto implica, dotar a las entidades subnacionales de mayores facultades de decisión respecto del conjunto de materias que inciden en el desarrollo urbano, no solo las referidas a las construcciones y sus usos, como sucede actualmente, sino también, las referidas a sistemas de transporte, infraestructura, redes de servicios e instalaciones, manejo integrado de residuos, gestión de tributos asociados a obras o actividades locales, entre otras.

El principal mecanismo para que la integración se produzca será que todas las intervenciones sobre las ciudades y el territorio, queden sujetas a su concordancia con un sistema que se debe alcanzar a través de una "planificación gobernada" por medio 
de un sistema descentralizado de decisiones urbanas y territoriales, lo cual implica, que las decisiones radicarán por defecto en el nivel local, y sólo pasarán a niveles superiores cuando la escala de la materia lo amerite o a nivel local no sea posible asumirlas.

En específico, el rol que se le asigna a los niveles subnacionales está contemplado en a lo menos tres dimensiones. El primero, a nivel municipal, se identifica en el que dice relación con la Integración Social y se plantea como principal objetivo general "revertir las actuales situaciones de segregación social urbana" (Giménez y Ugarte, 2014). Se indica que la municipalidad estará a cargo de la planificación, gestión comunal y toma de decisiones respecto de proyectos y obras de carácter comunal. Tendrá mayores atribuciones, que las actuales en materia de desarrollo urbano, conforme a las nuevas materias de los Instrumentos de Planificación Territorial que quedarían bajo su tuición (Giménez y Ugarte, 2014).

En cuanto a la Escala Regional, será competencia del gobierno regional, tendrá atribuciones sobre la planificación y gestión territorial y sobre las intervenciones así como proyectos de alcance regional. También, se encargará de la planificación urbana intercomunal y de las obras de carácter regional o intercomunal, junto con prestar soporte y asesoría técnica para la generación de proyectos y la planificación urbana de las ciudades, pueblos y localidades rurales que lo requieran (Giménez y Ugarte, 2014).

A Escala Nacional, estará encabezada por un Ministerio de Ciudades, Vivienda y Desarrollo Territorial. Será el encargado de la fijación de reglas y la formulación de políticas nacionales sobre planificación y gestión urbana y territorial, sobre instalaciones de infraestructura, además de proyectos u obras de carácter estratégico o de importancia nacional establecidos por ley. Le corresponderá también la supervisión del cumplimiento de las reglas de planificación en los instrumentos de los demás niveles, además de decidir sobre el financiamiento e implementación de las obras públicas de importancia o alcance nacional.

\section{El contraste empírico y la opinión de expertos. Las nuevas políticas urbanas de planificación territorial}

Los resultados corresponden a un estudio liderado por el Centro de Sociología y Políticas Locales de la Universidad Pablo de Olavide y se aplicó en 5 países de América Latina (acá se muestra sólo los resultados para el caso de Chile). El objetivo del estudio fue conocer la lógica de la evaluación de políticas públicas en los procesos de planificación territorial, que permita analizar la calidad del diseño de las políticas urbanas, con el objeto de garantizar su evaluabilidad, racionalidad y coherencia ex-ante a su implementación. En esta sección se muestra la opinión de los expertos consultados en Chile ${ }^{(2)}$, sobre su visión de las políticas urbanas e instrumentos de planificación territorial y a la participación e influencia de distintos actores institucionales y sociales en su diseño e implementación.

En primer lugar, se consultó sobre el carácter que tienen los objetivos e instrumentos de planificación de desarrollo urbano y/o territorial. En concreto, la escala utilizada fue de recorrido 0-10 para conocer el carácter de los objetivos de la planificación urbana. El punto 0 representaba objetivos totalmente sectoriales y el punto 10 representaba políticas y objetivos totalmente integrales, articulados o comprehensivos.

El promedio para el caso de los consultados en Chile se sitúa en 3,41 puntos, lo cual significa, que en general y a juicio de los expertos consultados, los objetivos de dichos instrumentos y políticas siguen siendo percibidos más cerca de ser sectoriales que integrales. Sólo el 9,8\% de las personas consultadas consideran que los objetivos contenidos en las políticas e instrumentos de planificación, son comprehensivos o integrales, siendo los más relevantes los orientados al desarrollo económico $(48,8 \%)$ y el urbanismo $(36,6 \%)$.

Frente a la pregunta ¿Qué objetivos consideran más relevantes para la promoción del desarrollo urbano? Para el caso de las grandes ciudades (tal como se aprecia en 
la Tabla 1), que son espacios urbanos que representan una mayor concentración de población y relacionadas con las áreas metropolitanas en Chile, se destacan la Movilidad Urbana, tales como transporte público, nuevas formas de movilidad (76,9\%), Calidad Ambiental del aíre y ruidos $(71,8 \%)$;
Infraestructuras y servicios urbanos básicos, agua, residuos, energía (61,5\%). Por su parte, las menores frecuencias tienen relación con inclusión intercultural (minorías, inmigrantes, refugiados), atracción de talento e igualdad de género.

\section{Tabla 1}

Los objetivos del desarrollo urbano: Porcentajes sobre el total de casos

\begin{tabular}{lcc}
\multicolumn{1}{c}{ Objetivos } & Grandes ciudades & Ciudades medias \\
\hline $\begin{array}{l}\text { Infraestructuras y servicios urbanos básicos (agua, residuos, } \\
\text { energía, ...) }\end{array}$ & $\mathbf{6 1 , 5}$ & $\mathbf{8 7 , 2}$ \\
Capacitación capital humano, formación, & 35,9 & 43,6 \\
Inclusión intercultural (minorías, inmigrantes, refugiados,..) & 7,7 & 12,8 \\
Calidad ambiental (del aire, ruidos,...) & $\mathbf{7 1 , 8}$ & 28,2 \\
Cohesión y convivencia vecinal & 20,5 & 38,5 \\
Preservación patrimonio cultural y natural & 30,8 & 38,5 \\
Capital social y vida asociativa & 15,4 & 38,5 \\
$\begin{array}{l}\text { Movilidad urbana (transporte público, nuevas formas de } \\
\text { movilidad,...) } \\
\text { Pobreza y desigualdad, servicios de salud, educación e integración } \\
\text { social }\end{array}$ Transición energética, producción/consumo responsables & $\mathbf{7 6 , 9}$ & 33,3 \\
(economía circular,..) & 56,4 & $\mathbf{6 6 , 7}$ \\
Igualdad de género & 46,2 & 15,4 \\
Atracción de talento y actividades creativas & 12,8 & 20,5 \\
Acceso a la vivienda & 10,3 & 25,6 \\
Economía local (incentivos/ayudas a empresas, emprendimiento) & 25,6 & 33,3 \\
Participación ciudadana en la vida social y política & 15,4 & $\mathbf{6 1 , 5}$
\end{tabular}

Fuente: Elaboración propia, 2020.

En cuanto a las ciudades medias, que representa el espacio urbano donde habita la mayor cantidad de población de regiones no vinculadas a áreas metropolitanas, se aprecia que los objetivos que consideran más relevantes son los relacionados con infraestructura básica y conectividad (agua, caminos, electrificación) alcanzando un 87,2\% de las menciones en los consultados (ver Tabla 1). Esto se puede explicar a las necesidades que existen en territorios aún por la escasez de agua y la tasa de kilómetros de caminos versus los caminos pavimentados. Por ejemplo, en la región de Los Ríos, del 100\% de caminos potencialmente pavimentables sólo se tiene una cifra cercana al 40\%. En segundo lugar, 
aparecen los objetivos relacionados con Pobreza y Desigualdad con un $66,7 \%$ y Economía Local con un $61,5 \%$.

Respecto a las estrategias más adecuadas para fomentar el desarrollo urbano integral y sostenible, y tal como se puede apreciar en la Tabla 2, en las grandes ciudades el orden de preferencias es el siguiente:
Primero, desarrollo de políticas explícitas para fomentar el desarrollo urbano sostenible (76,9\%); segundo, el desarrollo de políticas explícitas que promuevan un enfoque integral $(64,1 \%)$; tercero, potenciar la participación de la ciudadanía y las asociaciones locales en su diseño e implementación (61,5\%).

Tabla 2

Las estrategias para el fomento del desarrollo urbano: Porcentajes sobre total de casos

\begin{tabular}{|c|c|c|}
\hline Estrategias & Grandes ciudades & Ciudades medias \\
\hline Políticas para fomentar el desarrollo urbano sostenible & 76,9 & 66,7 \\
\hline $\begin{array}{l}\text { Sinergias entre políticas sectoriales que se desarrollan en el } \\
\text { mismo territorio }\end{array}$ & 35,9 & 35,9 \\
\hline Cooperación con agentes socio-económicos locales & 28,2 & 43,6 \\
\hline Asesoramiento a los municipios para iniciar proyectos & 23,1 & 53,8 \\
\hline $\begin{array}{l}\text { Cooperación entre departamentos y agencias sectoriales en los } \\
\text { municipios }\end{array}$ & 12,8 & 30,8 \\
\hline Participación de la ciudadanía y asociaciones de la sociedad civil & 61,5 & 79,5 \\
\hline $\begin{array}{l}\text { Gobiernos locales con suficientes capacidades políticas y } \\
\text { administrativas }\end{array}$ & 48,7 & 66,7 \\
\hline $\begin{array}{l}\text { Potenciar el desarrollo policéntrico (rural-urbano, áreas } \\
\text { metropolitanas,...) }\end{array}$ & 46,2 & 28,2 \\
\hline Formación en análisis y evaluación de políticas públicas & 25,6 & 25,6 \\
\hline $\begin{array}{l}\text { Desarrollo de un enfoque integral, comprehensivo del desarrollo } \\
\text { urbano }\end{array}$ & 64,1 & 30,8 \\
\hline $\begin{array}{l}\text { Cooperación entre administraciones (nacional, estatal, federal, } \\
\text { municipal,...) }\end{array}$ & 51,3 & 35,9 \\
\hline Cooperación/redes de intercambio y aprendizaje entre municipios & 15,4 & 30,8 \\
\hline
\end{tabular}

Fuente: Elaboración propia, 2020.

Por su parte, en las ciudades intermedias el primer lugar lo obtiene el desarrollo de estrategias de participación ciudadana y asociaciones locales con un 79,5\% del total de las menciones; en segundo lugar, el desarrollo de políticas explícitas para fomentar el desarrollo sostenible (66,7\%), compartido con gobiernos locales con capacidades políticas y administrativas con un $66,7 \%$.

Como contrapartida, las estrategias menos mencionadas en las grandes ciudades fueron; asesoramiento a municipios para iniciar proyectos $(23,1 \%)$; cooperación, intercambio y aprendizaje entre municipios $(15,4 \%) ; y$, cooperación de agencias sectoriales a nivel municipal con un $12,8 \%$. Por su parte, en las ciudades intermedias, las estrategias que se perciben menos valoradas son la formación en análisis y evaluación de políticas públicas $(25,6 \%)$; potenciar el desarrollo 
policéntrico con un 28,2\% (rural-urbano, áreas metropolitanas) y compartido con un $30,8 \%$ se encuentra cooperación/redes de intercambio, aprendizaje entre municipios; $\mathrm{y}$, desarrollo de un enfoque integral y cooperación entre sectores y gobiernos regionales.

Respecto a la influencia de actores e instituciones en el diseño (elaboración) y ejecución (implementación) de políticas e instrumentos de desarrollo y planificación urbano/territorial, se puede indicar lo siguiente. De acuerdo con los expertos, y tal como se puede apreciar en la Tabla 3, coinciden en que el actor más influyente en el diseño es el Gobierno Nacional, a través de sus ministerios (media igual 7,97).

Tabla 3

Actores influyentes en el diseño de políticas e instrumentos de desarrollo urbano en su país

\begin{tabular}{lc}
\multicolumn{1}{c}{ Actor } & Valor \\
\hline Gobiernos nacionales/federales & 7,97 \\
Actores del sector privado (empresas) & 6,15 \\
Gobiernos regionales/provinciales & 4,10 \\
Representantes del gobierno local (municipios, municipalidades,..) & 3,97 \\
Actores de la sociedad civil (asociaciones, ONGs, ciudadanía) & 3,10 \\
Miembros de la administración local (funcionarios, técnicos,...) & 2,97 \\
\hline
\end{tabular}

Nota: 0: poco influyente vs. 10: el más influyente

Fuente: Elaboración propia, 2020.

Lo sigue el sector privado a través de empresas o grupos empresariales (media igual a 6,15) que ejercen presión para que sus intereses se reflejen en determinadas políticas y estrategias; y en tercer lugar, para el caso Chileno son los Gobiernos Regionales (media igual a 4,10), tal como se observa en la Tabla 3. Los menos influyentes en la etapa de diseño son los miembros de la administración local (media igual a 2,97), los Actores de la Sociedad Civil, ONG, ciudadanía en general (media igual a 3,10) y los representantes políticos de las municipalidades (media igual a 3,97).

Llama la atención la visión que existe respecto de la influencia de empresas en la etapa de diseño de proyectos urbanos, lo cual se puede explicar, por la relación que en los últimos 15 años en Chile se ha evidenciado con mayor fuerza entre la política y el sector privado (sobre todo en financiamiento irregular de campañas electorales), cuestiones que se han ido regulando a través de distintas normativas (ley de lobby, ley de transparencia, ley de regulación de financiamiento de campañas) para evitar la cooptación de autoridades, especialmente, cuando se trata de tomar decisiones sobre proyectos inmobiliarios o de otra naturaleza pero de gran envergadura.

En la etapa de la ejecución, el orden de influencia es el mismo pero con distinta ponderación, para el Gobierno Nacional (media igual a 7,41); para Actores del Sector Privado/Empresarial (media igual a 6,05), para los Gobiernos Regionales (media igual a $5,18)$, tal como se aprecia en la Tabla 4. Luego 
aparecen los representantes de gobiernos locales con una importancia media de 5,10. De acuerdo con los encuestados, nuevamente los menos influyentes en la ejecución son los miembros de las administraciones locales (media de 4,05) y los actores de la sociedad civil (media de 3,33).

Tabla 4

\section{Actores influyentes en la ejecución de políticas e instrumentos de desarrollo} urbano en su país

\begin{tabular}{lc}
\multicolumn{1}{c}{ Actor } & Valor \\
\hline Gobiernos nacionales/federales & 7,41 \\
Actores del sector privado (empresas) & 6,05 \\
Gobiernos regionales/provinciales & 5,18 \\
Representantes del gobierno local (municipios, municipalidades,...) & 5,10 \\
Miembros de la administración local (funcionarios, técnicos,...) & 4,05 \\
Actores de la sociedad civil (asociaciones, Ongs, ciudadanía) & 3,33 \\
\hline
\end{tabular}

Nota: 0: poco influyente vs. 10: el más influyente

Fuente: Elaboración propia, 2020.

Respecto a esta dimensión, cabe destacar la escasa valoración e importancia que en la práctica los expertos constatan que se le asigna a la participación ciudadana y a las autoridades locales, tanto en el diseño como en la ejecución de políticas e instrumentos de desarrollo urbano. Esta situación, ya ha sido evidenciada en otros trabajos (Montecinos 2006; Delamaza, 2011; Delamaza y Thayer, 2016) y se aprecia como una consecuencia propia del centralismo del país, y de la influencia de elites corporativas y gremiales en los grandes proyectos, desplazando a gobiernos locales, ciudadanía y sus organizaciones de algunos proyectos emblemáticos de planificación urbana.

No obstante aquello, se puede apreciar una irrupción de la participación ciudadana en la planificación de la gestión territorial, ya sea por legislaciones que la promueven de manera no vinculante como la ley de participación ciudadana $\mathrm{N}^{\circ} 20.500$, o por impulsos que le han dado los distintos gobiernos a la temática (Contreras y Montecinos, 2019). Otra razón fundamental para ello, ha sido por la irrupción de la propia sociedad civil organizada en distintas iniciativas de desarrollo urbano, pero que encuentran barreras burocráticas y rígidos diseños institucionales que impiden superar la etapa informativa y pasar a una etapa de mayor control social. Esto se puede ratificar en los siguientes casos emblemáticos desarrollados en la región de Los Ríos ${ }^{(3)}$ y que se tomaron como referencia empírica en el marco del estudio señalado anteriormente.

\subsection{Las iniciativas y proyectos de desarrollo urbano: Los casos del puente Cochrane y el Parque Urbano Catrico}

En esta sección se describe y analiza el desarrollo de dos proyectos emblemáticos para la región de Los Ríos de alto impacto urbano como son el Parque Urbano Catrico y el Puente Los Pelúes. Ambos proyectos 
tienen orígenes distintos, uno bottom up (en la comunidad) y otro top down (a nivel nacional sectorial), pero en su implementación presentan similares problemáticas relacionadas con la participación de la ciudadanía y de los gobiernos locales.

El Parque Urbano Catrico es un proyecto de intervención y restauración urbana, que nace desde los pobladores y agrupaciones sociales en conjunto con el Gobierno Regional de Los Ríos, con el objetivo de encausar las necesidades de esparcimiento, seguridad y conservación de espacios públicos así como mejorar la calidad de vida para el sector sur de la ciudad de Valdivia, capital de la región de Los Ríos.

Contempla una intervención urbana de más de 65 mil metros cuadrados, los que hasta antes de esa fecha eran usados en una parte importante como sitio eriazo. Durante el año 2013, se comenzó a diseñar y construir este proyecto gracias a la iniciativa de pobladores $\mathrm{y}$ juntas de vecinos, quienes querían contar con un pulmón verde que sirviera de encuentro comunitario y espacio deportivo para la ciudadanía, y que a su vez, permitiera frenar los micro basurales del sector, los focos de delincuencia y el tráfico de drogas.

Los actores sociales, locales y regionales, desde su origen han sido protagonistas en el diseño y ejecución del proyecto, siendo ellos quienes plantearon un modelo de gobernanza para la puesta en marcha y la administración futura de la iniciativa. Las agrupaciones líderes de esta iniciativa, proponen un modelo que contempla una auto-administración gestionada por una corporación que diseñe, elabore y ponga en marcha acciones para sostener al parque de manera económica, medioambiental y socialmente sustentable.

A su vez, se contempla la autorregulación o fiscalización ciudadana. En esta última propuesta, es donde se ha encontrado la principal dificultad para su implementación, dado que a 3 años de finalización de su última etapa, aún no existe un acuerdo del Gobierno central con la comunidad para la administración de este proyecto. Sigue predominando la lógica ministerial y nacional de imponer un modelo de administración, que en este caso le asigna esa función al nivel municipal y no a la comunidad. Esto es sensible, dado que el municipio, y en su momento el gobierno regional, apoyan la propuesta de la comunidad, pero los niveles centrales de Gobierno (encargados de financiar la obra) no lo hacen, fundamentalmente, porque no tienen la facultad legal para entregar a la comunidad su administración.

En la actualidad, el cuidado de los parques corresponde a las municipalidades, quienes se encargan de la mantención de las áreas verdes y de la reparación de artefactos menores. Sin embargo, no contempla gestionar las actividades que se puedan suscitar dentro del recinto como las deportivas o culturales y que en términos prácticos dan sentido a un parque urbano. Acciones, que sí estarían contempladas en el nuevo Programa de Conservación de Parques Urbanos, recién iniciado en 2015, pero que no incluyen a la ciudadanía para administrar estos bienes públicos. Eso hace que esta iniciativa sea única en el país en cuanto a participación ciudadana, pero que no ha encontrado el diseño institucional adecuado para su implementación y conservación, especialmente, posterior al término del proyecto.

No cabe duda, que este proyecto representa el espíritu del nuevo enfoque de políticas públicas urbanas, pero carece de un espacio a la comunidad en la administración posterior a la ejecución de las obras. Es necesario, colocar atención en el transcurso de este proyecto que finaliza en tres años, especialmente, con los esfuerzos que se realicen para modificar el diseño institucional que permita incorporar a la comunidad en la administración de obras de alto impacto urbano, que además, surgieron a raíz de peticiones de la propia comunidad.

Un caso distinto es el proyecto Puente Cochrane (también conocido como Puente Los Pelúes). Esta iniciativa conectará la calle Cochrane de la ciudad de Valdivia con la calle Los Pelúes en el sector de Isla Teja de la misma ciudad, separadas por el cauce del rio principal de la misma. Comenzó su proceso en el año 
2014 y debido a diversos inconvenientes aún se encuentra en etapa de diseño, el cual se ha visto retrasado luego que en enero del año 2020 se paralizara nuevamente el proceso de Evaluación de Impacto Ambiental, tras la orden del Servicio de Evaluación Ambiental (SEA) de realizar una nueva consulta ciudadana a la comunidad.

En este proyecto, igualmente se ha impuesto una lógica top down en su diseño y ejecución, con una visión sectorial, sin participación ciudadana, ni del gobierno local, salvo en el nivel informativo y la que obliga la ley en la evaluación ambiental. La iniciativa está sometida desde abril del 2019 a evaluación ambiental y en diciembre de ese mismo año el Servicio de Vivienda y Urbanismo (órgano nacional con representación regional) presentó las respuestas para responder a las observaciones que se hicieron en el Estudio de Impacto Ambiental. Sin embargo, el SEA resolvió que las respuestas mostraban que el proyecto tiene aclaraciones, rectificaciones y ampliaciones que lo modifican sustancialmente, determinando aplicar una nueva consulta ciudadana, lo que ha retrasado el inicio del proyecto.

El puente, contaría con 325 metros de largo y 17 de ancho, sostenido con cuatro cepas que permitirían el tránsito fluvial. Este proyecto, que forma parte de la planificación del Ministerio de Vivienda y Urbanismo, en la medida que su diseño ha ido avanzando desde el año 2014, ha demostrado su complejidad y ha tenido que ir sumando al Municipio Local, al Gobierno Regional y a otros Ministerios (como el de Obras Públicas) con la finalidad de poder conseguir su autorización para la ejecución de la obra.

Básicamente la sumatoria de estos actores no ha sido producto de una actuación o planificación multinivel de los organismos públicos involucrados, muy por el contrario, esa estrategia ha sido utilizada a raíz de conflictos o crisis provocadas con la comunidad por el poco avance que demostraba el proyecto, el cual es una necesidad urgente para la ciudad, dada la alta congestión vehicular que se provoca en el único puente que conecta a la ciudad con la Isla Teja.

Si bien es cierto, para la comunidad local es de suma relevancia contar con esta obra, dado que facilita y mejora la conectividad de la ciudad, no han sido partícipes de su diseño, sino más bien han tenido que incorporarse porque otro servicio público (como el de Evaluación Ambiental) ha obligado que así sea. Acá se está en presencia de un proyecto urbano que ha tenido que incorporar a la sociedad civil de manera reactiva, retrasando más de la cuenta la ejecución de la misma obra.

\section{Conclusiones}

Las iniciativas de desarrollo urbano cuentan con un marco orientador sobre el diseño y la implementación de instrumentos de planificación territorial, a partir de la promulgación de la Política Nacional de Desarrollo Urbano en el año 2014. En ella se puede reflejar la necesidad de realizar esfuerzos institucionales por articular distintos niveles de gobierno, sobre todo, cuando las iniciativas son de alto impacto urbano, o requieren de múltiples intervenciones tanto en su diseño como en su implementación.

Antes de esa fecha, no era tradicional encontrar políticas públicas de planificación territorial que promuevan la articulación multinivel en el diseño de las iniciativas, sino más bien predominaba el paradigma del diseño vertical y reactivo. Es decir, sólo se sumaban a otros niveles de gobierno o actores de la sociedad civil, cuando existían conflictos entre instituciones o con organizaciones de la sociedad civil, por la intervención que implicaba un determinado proyecto. Generalmente, en la etapa de implementación surgían los inconvenientes, dado que no se consideraban aspectos de índole territorial que resultaban relevantes para la correcta ejecución de dichas iniciativas.

Pero la opinión de expertos y la evidencia expuesta en los casos descritos, indican que aún es escasa la articulación multinivel usada como estrategia de fomento del desarrollo urbano, para la ejecución de proyectos e 
instrumentos de planificación territorial. El modelo predominante sigue siendo sectorial, no se observa un modelo integral/holístico, tanto en el diagnóstico, definición de objetivos, intervención y ejecución de los proyectos. La participación de actores de la sociedad civil y de gobierno local es también escasa.

Claramente, se puede apreciar un contraste entre lo que sostiene la política nacional de desarrollo urbano y lo que opinan o perciben los expertos entrevistados, cuestión que también se ve reflejada en los casos de proyectos emblemáticos de desarrollo urbano que se encuentran en pleno desarrollo en la región de Los Ríos: Parque Urbano y Puente Los Pelúes (últimamente llamado Puente Cochrane).

De este modo, se podría señalar que la política nacional de desarrollo urbano, ha contribuido con entregar definiciones estratégicas, pero no logra tener incidencia en la práctica de la planificación territorial. Es decir, persiste la debilidad institucional de transformar el discurso multinivel en gestión multinivel, o transformar el discurso bottomup en capacidad de incidir de los gobiernos locales y la sociedad civil en el diseño y ejecución de políticas y proyectos de impacto urbano.

Es mucho mayor el impacto que provocan proyectos de alta complejidad urbana, cuando se impulsan sin considerar la participación ciudadana o la opinión de gobiernos locales. La promulgación de esta política y las correctas orientaciones estratégicas que contiene, no resuelve por sí sola esta forma tradicional de diseño y ejecución, sino va acompañada de modificación de los diseños institucionales en la coordinación y relación multinivel de gobierno.

Dos son los factores que pueden permitir mejorar esta situación. El primero, se refiere a perfeccionar los diseños institucionales para que incentiven la cooperación multinivel y multiactoral en la ejecución de proyectos orientados al desarrollo urbano. En la medida que se reduzca la excesiva dependencia jerárquica, fiscal y administrativa existente entre niveles de gobierno para implementar los instrumentos de planificación local/ regional, se puede reducir la lógica de gestión top down predominante en Chile. Para cumplir los propósitos de la panificación territorial, necesariamente debe existir coordinación y colaboración, pero también mayor descentralización hacia los gobiernos subnacionales de la capacidad de gasto y decisión fiscal sobre recursos públicos sectoriales.

Hoy no sucede, porque se imponen lógicas sectorialistas que no contemplan procesos de articulación, participación y de gobernanza como estrategias fundamentales para su ejecución. Urge considerar en el modelo de gobierno local y de planificación, mecanismos formales de coordinación de los instrumentos de planificación que se implementan en un mismo territorio. Se estima que en una comuna promedio en Chile, conviven a lo menos 10 instrumentos de planificación, los cuales hoy no cuentan con espacios formales de articulación y coordinación, pudiendo racionalmente ser el municipio el órgano que lo haga, se ve impedido porque del nivel sectorial y regional, no existe un reconocimiento legal para que ello suceda.

Un segundo factor relevante, se refiere a hacer vinculante la participación ciudadana en los instrumentos de planificación territorial. En la actualidad, no todos los proyectos con impacto urbano se someten a procesos de participación ciudadana, solo lo hacen aquellos que ingresan por el Sistema de Evaluación Ambiental y que se supone generarán impactos en el medio ambiente. Es más, existe la noción que es una estrategia que retrasa más que favorece la inversión rápida de proyectos en el territorio, por ello es que muchas veces se prefiere evadir el ingreso de algunas inversiones a este sistema.

Por otra parte, la legislación que promueve la participación ciudadana en la gestión pública carece de fuerza vinculante, dado que no asocia participación con decisión, sino que sólo la considera en su dimensión informativa y consultiva, provocando en las organizaciones de la sociedad civil desgano 
y escasa utilidad de participar en los procesos donde se les convoca a participar.

Las nociones que contempla la política de desarrollo urbano representan una oportunidad para avanzar en perfeccionar el sistema de gobierno subnacional y el de planificación urbana y territorial, pero se requiere un paso adicional para asignarle un rol más protagónico a las organizaciones de la sociedad civil y a la ciudadanía en general, en la construcción de su desarrollo de abajo hacia arriba. Ese paso tiene relación con transformar la política de desarrollo urbano en instrumentos vinculantes con los principios que sostiene, de tal forma, que el entramado burocrático e institucional encuentre los incentivos necesarios para adaptarse a los requerimientos de este nuevo esquema de políticas públicas de desarrollo urbano.

\section{Notas}

1 Proyecto coordinado por el Dr. Clemente Navarro del Centro de Sociología y Políticas Locales de la Universidad Pablo de Olavide donde el Centro de Estudios Regionales de la Universidad Austral de Chile sirvió de contraparte colaboradora para proveer información sobre Chile. La metodología de investigación fue cuali-cuantitativa. Se aplicó encuesta a informantes expertos y líderes sociales, análisis de frecuencia y contenido. Los resultados del análisis de esta recogida de datos se contrastaron con casos emblemáticos que sirvieron como experiencias ejemplificadoras del fenómeno estudiado. El objetivo del estudio fue conocer la lógica de la evaluación de políticas públicas en los procesos de planificación territorial. La entidad financiadora es la Junta de Andalucía y fue desarrollado en el marco de la convocatoria para la concesión de subvenciones, en régimen de concurrencia competitiva, para el desarrollo de proyectos de cooperación internacional en las líneas de actuación de fomento y formación en las materias de competencia de la Consejería de Fomento, Infraestructuras y Ordenación del Territorio, con objeto de promover el desarrollo de proyectos de cooperación internacional realizados por las Universidades Públicas Andaluzas.

${ }^{2}$ Expertos académicos y líderes sociales y técnicos vinculados a instituciones públicas de nivel nacional y subnacional.

${ }^{3}$ En el marco del desarrollo del estudio donde participó como contraparte el Centro de Estudios Regionales de la Universidad Austral, se seleccionaron dos proyectos de desarrollo urbano que tuvieran algún grado de relevancia regional por distintos gobiernos desde hace a los menos 10 años en su avance y desarrollo. Estos casos en su implementación práctica demuestran la importancia de la articulación multinivel y la participación de actores locales en su desarrollo.

\section{Referencias bibliográficas}

Alencastro, A. P., Castañón, J., Quiñonez, M. R., y Egas, F. (2020). Planificación estratégica para el desarrollo territorial de la Provincia Esmeraldas en Ecuador. Revista de Ciencias Sociales (Ve), XXVI(3), 130-147. https://doi. org/10.31876/rcs.v26i3.33238

Contreras, P., y Montecinos, E. (2019). Democracia y participación ciudadana: Tipología y mecanismos para la implementación. Revista de Ciencias Sociales (Ve), XXV(1), 178-191.

De La Fuente, G., y Mlynarz, D. (Eds.) (2013). El pueblo unido... Mitos y realidades sobre la participación ciudadana en Chile. Editorial Alberto Hurtado.

Delamaza, G. (2011). Espacio público y participación ciudadana en la gestión pública en Chile: límites y posibilidades. Polis, 10(30), 45-75.

Delamaza, G. y Thayer, L. E. (2016). Percepciones políticas y prácticas de participación como instrumento 
para la gobernanza de los territorios. EURE, 42(127), 137-158. http://dx.doi.org/10.4067/S025071612016000300006

García, J. R., Aldape, L. A., y Esquivel, F. A. (2020). Perspectivas del desarrollo social y rural en México. Revista de Ciencias Sociales (Ve), XXVI(3), 45-55. https://doi.org/10.31876/rcs. v26i3.33230

Giménez, P., y Ugarte, J. (2014). Hacia una nueva política urbana para Chile. Política Nacional de Desarrollo Urbano. PNUD y Ministerio de Vivienda y Urbanismo (MINVU). http://cndu.gob.cl/wp-content/ uploads/2014/10/L4-PoliticaNacionalUrbana.pdf

Grin, J. E., Completa, E. R., Abrucio, F. L., y Carrera-Hernández, A. P. (2019). Capacidades estatales de los gobiernos locales iberoamericanos: Actualidad, brechas y perspectivas. Editora FGV.

Gutiérrez, J. J. (2017). México y la Nueva Agenda Urbana. Hoja de ruta con trazos invisibles. Bitácora Urbano Territorial, 27(2), 35-43. http://dx.doi. org/10.15446/bitacora.v27n2.63133

Irarrázaval, I., Aninat, I., Rodríguez, J., y Razmilic, S. (2020). Más allá de Santiago: Descentralización fiscal en Chile. Centro de Estudios Públicos (CEP) y el Centro de Políticas Públicas UC.

Livert, F., Gainza, X., y Mogollón, R. (2018). Factores políticos en la distribución de inversión pública: Problemas de planificación y gobernanza en Chile. Sociedad Chilena de Políticas Públicas. https://www. sociedadpoliticaspublicas.cl/archivos/ noveno/Politica Livert Felipe.pdf

Montecinos, E. (2005). Antecedentes sobre la relación histórica centralismo y descentralización en Chile. Revista Venezolana de Gerencia, 10(31), 443 - 462.

Montecinos, E. (2006). Descentralización y Democracia en Chile: Análisis sobre la participación ciudadana en el presupuesto participativo y el plan de desarrollo comunal. Revista de Ciencia Política, 26(2), 191-208.

Montecinos, E. (2020). Elección de gobernadores regionales en Chile: Escenarios de cambio en las relaciones intergubernamentales. Revista de Ciencia Política, 40(3), 567-587. https://doi.org/10.4067/S0718090X2020005000101

Navarro, C. J., Rodríguez-García, M. J., y Ramírez-Gómez, I. (2018). La agenda del desarrollo urbano integral en España (1994-2013). ANDULI, Revista Andaluza de Ciencias Sociales, 17, 47-66. http://dx.doi.org/10.12795/ anduli.2018.i17.03

Navarro, C. J., y Ramírez, M. A. (2000). Autonomía y gobierno municipal en perspectiva comparada. El caso de la Unión Europea y Latinoamérica. Revista de Humanidades, (11), 273287.

Navarro-Yáñez, C., y Rodríguez-García, M. (2020). Urban policies as multilevel policy mixes. The comparative urban portfolio analysis to study the strategies of integral urban development initiatives. Cities, 102, 102716.

Orellana, A., Vicuña, M., y Moris, R. (2017). Planificación urbana y calidad de vida: aproximación al estado de la planificación local en Chile. Cuadernos de Vivienda y urbanismo, 10(19), 88-109. https:// doi.org/10.11144/Javeriana.cvu1019.pucv

Organización para la Cooperación y el 
Desarrollo Económicos - OCDE (2017). National Urban Policy in OECD countries. OCDE. https:// dx.doi.org/10.1787/9789264271906en

Ortiz, E., y Valenzuela, E. (2018). Chile, un siglo de pugna por la democratización de las regiones. Representación minimizada y centralismo transversal. Revista de Estudios Políticos, 52, 3656.

Quispe, G., Ayaviri, D., y Maldonado, R. (2018). Participación de los actores en el desarrollo local en entornos rurales. Revista de Ciencias Sociales (Ve), XXIV(3), 62-82.

Rodríguez-García, M. J., y Navarro, C. J. (2016). Gobernanza local comparada: el análisis de los patrones de influencia en los sistemas políticos locales. REIS: Revista Española de Investigaciones Sociológicas, 13, 127-142. http:// dx.doi.org/10.5477/cis/reis.153.127

Ruano,J.M.,yVial,M.C.(Eds.)(2016).Manual de gobiernos locales en Iberoamérica. CLAD. https://ichem.uautonoma. cl/wp-content/uploads/2016/12/ MANUALgobLocalesCLAD-1.pdf

UN-Habitat (2014). The evolution of National Urban Policies - A global overview. UN-Habitat. https://unhabitat.org/theevolution-of-national-urban-policies

UN-Habitat (2017). The new Urban agenda. UN-Habitat. https://habitat3.org/thenew-urban-agenda/ 